Case Report

\title{
Bacteremia due to Leuconostoc pseudomesenteroides in a Patient with Acute Lymphoblastic Leukemia: Case Report and Review of the Literature
}

\author{
Kazuko Ino, ${ }^{1}$ Kazunori Nakase, ${ }^{1,2}$ Kei Suzuki, ${ }^{1,3}$ Akiko Nakamura, ${ }^{4}$ \\ Atsushi Fujieda, ${ }^{1}$ and Naoyuki Katayama ${ }^{1}$ \\ ${ }^{1}$ Department of Hematology and Oncology, Mie University Hospital, Tsu, Mie 514-8507, Japan \\ ${ }^{2}$ Cancer Center, Mie University Hospital, Tsu, Mie 514-8507, Japan \\ ${ }^{3}$ Emergency and Critical Care Center, Mie University Hospital, Tsu, Mie 514-8507, Japan \\ ${ }^{4}$ Central Clinical Laboratories, Mie University Hospital, Tsu, Mie 514-8507, Japan \\ Correspondence should be addressed to Kazunori Nakase; k2nakase@clin.medic.mie-u.ac.jp
}

Received 26 April 2016; Revised 21 July 2016; Accepted 28 July 2016

Academic Editor: Akimichi Ohsaka

Copyright ( 2016 Kazuko Ino et al. This is an open access article distributed under the Creative Commons Attribution License, which permits unrestricted use, distribution, and reproduction in any medium, provided the original work is properly cited.

Leuconostoc species are vancomycin-resistant Gram-positive cocci. Infections due to Leuconostoc species have been reported in various immunocompromised patients, but little is known about such infection in patients with hematologic malignancies. We report a case of Leuconostoc infection in a 44 -year-old woman with acute lymphoblastic leukemia. The patient developed a high fever despite antimicrobial therapy with doripenem after induction chemotherapy. After an isolate from blood cultures was identified as L. pseudomesenteroides, we changed the antibiotics to piperacillin-tazobactam and gentamicin, after which the patient recovered from the infection. Physicians should be aware of Leuconostoc species as causative pathogen if they encounter Gram-positive cocci bacteremia resistant to standard antibiotics such as vancomycin and teicoplanin, especially in patients with hematologic malignancies.

\section{Introduction}

Leuconostoc species are Gram-positive cocci (GPC) that naturally reside in dairy products, vegetables, and legumes and occasionally in the human vagina and stool samples [1]. The prominent characteristic of Leuconostoc species is general resistance to glycopeptides, such as vancomycin (VCM) and teicoplanin (TEIC); this resistance is due to the absence of a target for these agents in the cell wall [2]. Since the first case of Leuconostoc infection in human was reported in 1985 [3], a variety of infections due to these bacteria have been reported in immunocompromised patients $[1,3]$. However, little is known about this infection in patients with hematologic malignancies [4-6]. Here, we present a case of Leuconostoc bacteremia during chemotherapy in a patient with acute lymphoblastic leukemia (ALL) and review the literature describing its infection in hematologic patients.

\section{Case Report}

A 44-year-old woman was referred to our hospital because of a persistent fever and right-side back pain. On admission, her peripheral blood showed a hemoglobin level of $12.4 \mathrm{~g} /$ $\mathrm{dL}$, platelet count of $2.7 \times 10^{4} / \mu \mathrm{L}$, leukocyte count of 5,080/ $\mu \mathrm{L}$ with $17 \%$ abnormal lymphocytes, and an elevated lactate dehydrogenase level at $1,873 \mathrm{IU} / \mathrm{L}$. The bone marrow was hypercellular, with $92 \%$ of myeloperoxidase-negative blast cells expressing CD10, CD19, CD20, CD34, and TdT. Cytogenetic analysis showed a complex karyotype without $\mathrm{t}(9 ; 22)$. The patient was diagnosed with precursorB ALL and received induction chemotherapy comprising cyclophosphamide, doxorubicin, vincristine, L-asparaginase, and prednisolone (Figure 1). Since her admission (4 days before the initiation of chemotherapy), doripenem (DRPM) $(1 \mathrm{~g} \times 3$ /day) had been given because of a persistent fever 


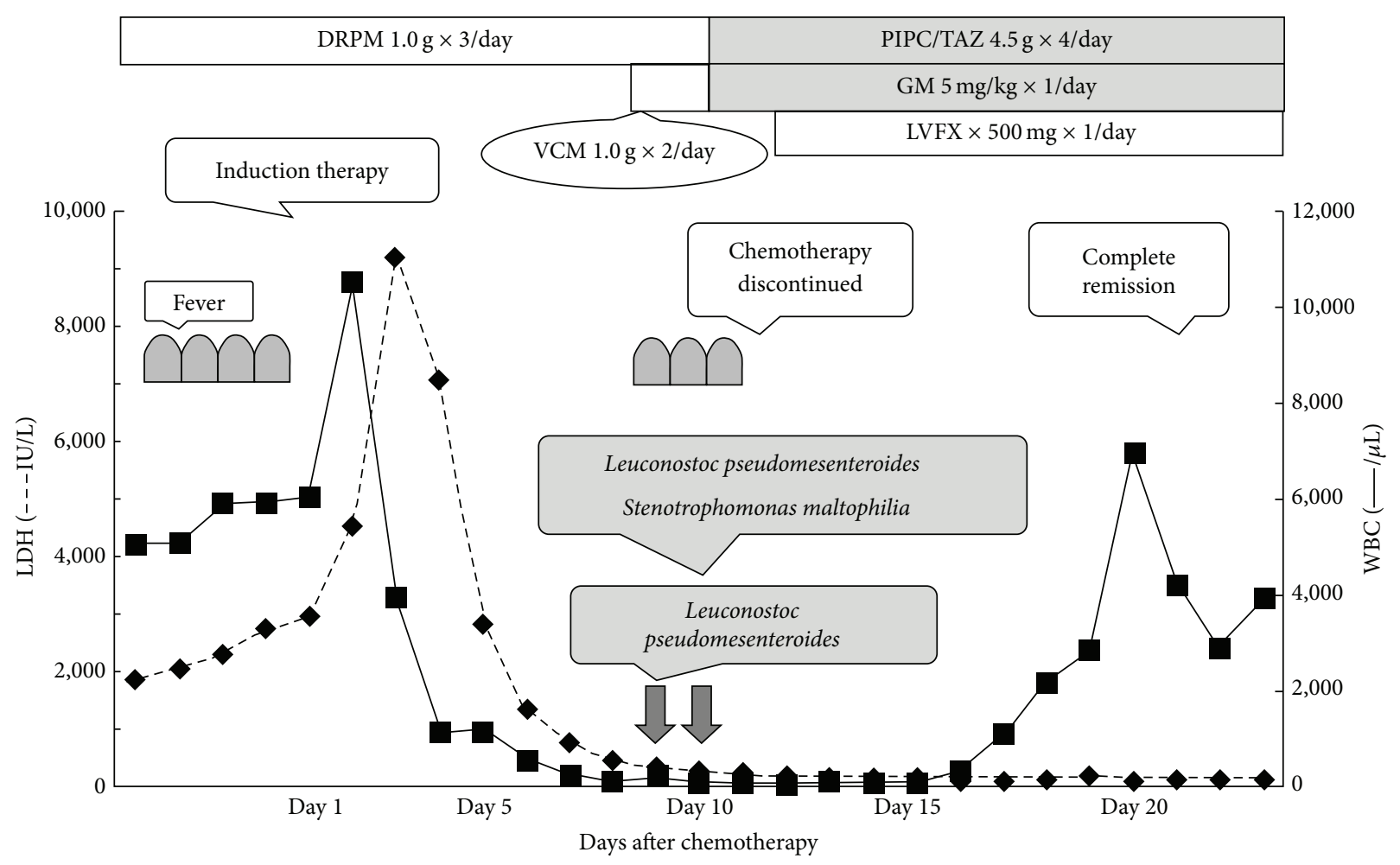

FIGURE 1: Clinical course of our case. Induction therapy consisted of cyclophosphamide $1,200 \mathrm{mg} / \mathrm{m}^{2}$ on day 1 , doxorubicine $60 \mathrm{mg} / \mathrm{m}^{2}$ on days $1-3$, vincristine $1.3 \mathrm{mg} / \mathrm{m}^{2}$ on days $1,8,15$, and 22 , L-asparaginase $3,000 \mathrm{U} / \mathrm{m}^{2}$ on days $9,11,13,16,18$, and 20 , and prednisolone $60 \mathrm{mg} / \mathrm{m}^{2}$ on days 1-21. DRPM, doripenem; VCM, vancomycin; PIPC/TAZ, piperacillin-tazobactam; GM, gentamicin; and LVFX, levofloxacin.

suspected to be caused by a bacterial infection. Although the fever subsided within a week, DRPM continued even after that. Fluconazole $(200 \mathrm{mg} \times 1 /$ day, oral $)$ was also used as antifungal prophylaxis. Five days after the initiation of chemotherapy (day 5$)$, she became neutropenic $(<500 / \mu \mathrm{L})$, and granulocyte colony stimulating factor (filgrastim, $300 \mu \mathrm{g}$ $\times 1 /$ day, drip infusion) therapy was started. However, she developed a high fever, and two sets of blood cultures were positive for GPC on day 9. Consequently, we commenced VCM administration immediately. As her menstrual period overlapped the duration of the chemotherapy, her vaginal bleeding persisted and was accompanied by endometritis, which seemed to be the portal of entry for the etiological pathogen. Two days later, however, the isolate was confirmed as L. pseudomesenteroides, which is naturally resistant to VCM. Meanwhile, no organisms were identified from the culture of vaginal discharge. Considering the neutropenic status, we changed the antibiotics to piperacillin-tazobactam $(4.5 \mathrm{~g}$ $\times 4 /$ day $)$ and gentamicin $(5 \mathrm{mg} / \mathrm{kg} \times 1 /$ day $)$ and removed the central venous catheter. Because the blood culture also identified Stenotrophomonas maltophilia on day 10, we also added levofloxacin $(500 \mathrm{mg} \times 1$ /day). After receiving these three antibiotics for one week, the bacteria were cleared from the circulation, and the patient became afebrile. During this period, she achieved complete remission. The antibiotics were administered for 2 weeks.
TABLE 1: Minimal inhibitory concentration of isolates.

\begin{tabular}{lc}
\hline Antibiotics & $\mu \mathrm{g} / \mathrm{mL}$ \\
\hline Penicillin G & 1 \\
Ampicillin & 2 \\
Cefotiam & $>4$ \\
Cefotaxime & $>4$ \\
Meropenem & $>2$ \\
Erythromycin & $<0.12$ \\
Clarithromycin & $<0.12$ \\
Vancomycin & $>1$ \\
Levofloxacin & 1 \\
Clindamycin & $<0.12$ \\
\hline
\end{tabular}

\section{Discussion}

GPC bacteremia is increasingly recognized in neutropenic patients with acute leukemia during chemotherapy because of the frequent use of central venous catheter, oral antibacterial prophylaxis with fluoroquinolone, and chemotherapyinduced disruption of the intestinal mucosa [7]. Several guidelines for febrile neutropenia recommend early empirical treatment with VCM if GPC are detected in the blood culture $[7,8]$. In our case, however, the VCM-resistant GPC, L. pseudomesenteroides was identified during the neutropenic period (Table 1). As TEIC susceptibility was not tested, the isolates' resistance to its agent was unclear. Nine cases of Leuconostoc 


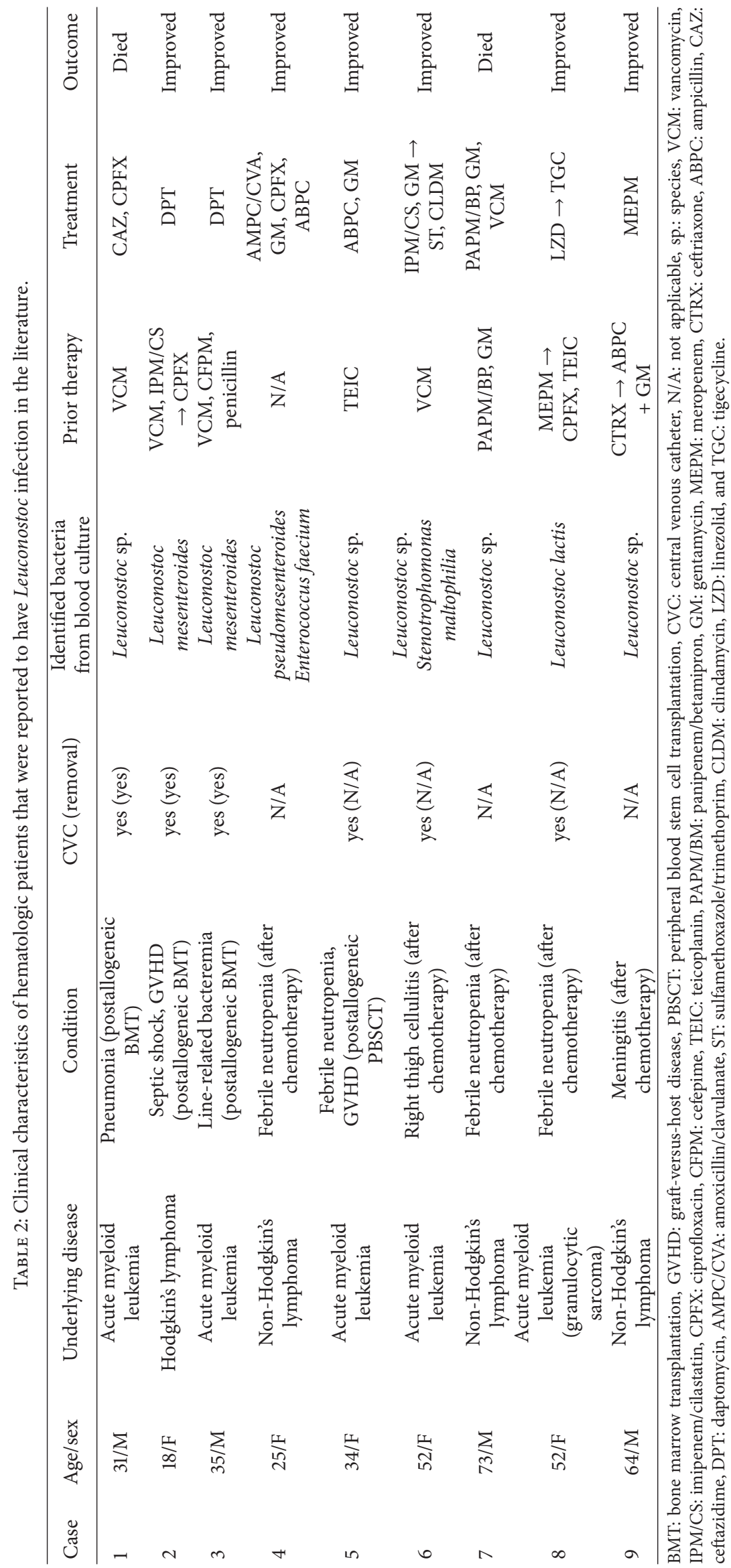


infection have been previously reported in patients with hematologic malignancy (Table 2) [4-6, 9-12], including five cases of acute myeloid leukemia, three cases of non-Hodgkin's lymphoma, and one case of Hodgkin's lymphoma. Our case is the first ALL patient to be successfully treated. Notably, two (cases 1 and 7 in Table 2) of the nine hematologic cases died owing to inappropriate use of antibiotics $[4,5]$, though only a few deaths related to Leuconostoc species have been described in nonhematologic patients [5]. In case 1 with acute myeloid leukemia [4], a Leuconostoc species was isolated from blood cultures during the treatment of methicillin-resistant Staphylococcus aureus pneumonia with VCM. However, the organism was regarded as a contaminant of skin origin; thus VCM withdrawal was not justified because the patient was afebrile. As a result, the patient died from the progression of Leuconostoc infection. In case 7 with non-Hodgkin's lymphoma [5], the isolate was initially misdiagnosed as $\alpha$-Streptococcus, and postmortem reevaluation of the blood culture confirmed that the GPC was a Leuconostoc species. Therefore, it is important to diagnose precisely and to administer the appropriate antibiotic promptly after confirmation of Leuconostoc species even if the patient is in an afebrile condition.

Recommended antibiotics for Leuconostoc infection include penicillin $\mathrm{G}$, ampicillin, clindamycin, carbapenem, and/or aminoglycosides [13]. Consistent with our isolate showing a high minimum inhibitory concentration (MIC) to meropenem (Table 1), carbapenem-resistant isolates have also been described [14]. Recently, daptomycin has arisen as a promising agent for VCM-resistant GPC infection [9]. In the current patient, the susceptibility of the isolates to piperacillin-tazobactam and gentamicin could not be evaluated. Nevertheless, we selected these two agents under consideration of both anti-Pseudomonas and anti-Leuconostoc activities because the patient remained severely neutropenic. However, as the blood culture additionally yielded $S$. maltophilia, we also administered levofloxacin owing to a low MIC for this isolate. As levofloxacin had a low MIC for $L$. pseudomesenteroides, this agent might also exhibit an additive antibacterial effect for the Leuconostoc isolates.

In conclusion, VCM-resistant GPC should be considered as a causative pathogen in infections, even in patients with hematologic malignancy. Since Leuconostoc species are easily misidentified as Streptococcus, Enterococcus, or Lactobacillus $[4,5]$, it is particularly important to distinguish between these species. At present, proper management, including antibiotic therapy for this infection, has not yet been established. Accordingly, further accumulation of cases of Leuconostoc infection such as the present case is needed to better understand detailed clinical characteristics in patients with hematologic malignancies.

\section{Competing Interests}

The authors declare that there is no conflict of interests regarding the patient's data.

\section{References}

[1] S. Handwerger, H. Horowitz, K. Coburn, A. Kolokathis, and G. P. Wormser, "Infection due to Leuconostoc species: six cases and review," Reviews of Infectious Diseases, vol. 12, no. 4, pp. 602-610, 1990.

[2] K. S. Templin, T. Crook, T. Riley III, C. Whitener, and R. C. Aber, "Spontaneous bacterial peritonitis and bacteremia due to Leuconostoc species in a patient with end-stage liver disease: a case report," Journal of Infection, vol. 43, no. 2, pp. 155-157, 2001.

[3] A. Buu-Hoi, C. Branger, and J. F. Acar, "Vancomycin-resistant streptococci or Leuconostoc sp," Antimicrobial Agents and Chemotherapy, vol. 28, no. 3, pp. 458-460, 1985.

[4] P. Giraud, M. Attal, J. Lemouzy, F. Huguet, D. Schlaifer, and J. Pris, "Leuconostoc, a potential pathogen in bone marrow transplantation,” The Lancet, vol. 341, no. 8858, pp. 1481-1482, 1993.

[5] K. Ishiyama, H. Yamazaki, Y. Senda, H. Yamauchi, and S. Nakao, "Leuconostoc bacteremia in three patients with malignancies," Journal of Infection and Chemotherapy, vol. 17, no. 3, pp. 412418, 2011.

[6] H. Holik, B. Coha, M. Šiško, and M. Tomić-Paradžik, "Leuconostoc sp. meningitis in a patient treated with rituximab for mantle cell lymphoma," Turkish Journal of Hematology, vol. 32, no. 3, pp. 271-274, 2015.

[7] A. G. Freifeld, E. J. Bow, K. A. Sepkowitz et al., "Clinical practice guideline for the use of antimicrobial agents in neutropenic patients with cancer: 2010 Update by the Infectious Diseases Society of America," Clinical Infectious Diseases, vol. 52, no. 4, pp. e56-e93, 2011.

[8] K. Tamura, "Clinical guidelines for the management of neutropenic patients with unexplained fever in Japan: validation by the Japan Febrile Neutropenia Study Group," International Journal of Antimicrobial Agents, vol. 26, no. 2, pp. S123-S127, 2005.

[9] Y. Golan, D. D. Poutsiaka, S. Tozzi, S. Hadley, and D. R. Snydman, "Daptomycin for line-related Leuconostoc bacteraemia," Journal of Antimicrobial Chemotherapy, vol. 47, no. 3, pp. 364365, 2001.

[10] P. Svec, A. Sevcikova, I. Sedlacek, J. Bednarova, C. Snauwaert, and $\mathrm{K}$. Lefebvre, "Identification of lactic acid bacteria isolated from human blood cultures," FEMS Immunology and Medical Microbiology, vol. 49, pp. 192-196, 2007.

[11] R. Yamazaki, T. Mori, K. Sugita, Y. Aisa, Y. Ikeda, and S. Okamoto, "Leuconostoc septicemia in a neutropenic patient with acute myelogenous leukemia relapsed after allogeneic peripheral blood stem cell transplantation," Transplant Infectious Disease, vol. 11, no. 1, pp. 94-95, 2009.

[12] T. Patel, A. Molloy, R. Smith, and I. Balakrishnan, "Successful treatment of Leuconostoc bacteremia in a neutropenic patient with tigecycline," Infectious Disease Reports, vol. 4, no. 2, pp. 124-125, 2012.

[13] J. M. Swenson, R. R. Facklam, and C. Thornsberry, "Antimicrobial susceptibility of vancomycin-resistant Leuconostoc, Pediococcus, and Lactobacillus species," Antimicrobial Agents and Chemotherapy, vol. 34, no. 4, pp. 543-549, 1990.

[14] G. Deye, J. Lewis, J. Patterson, and J. Jorgensen, "A case of Leuconostoc ventriculitis with resistance to carbapenem antibiotics," Clinical Infectious Diseases, vol. 37, no. 6, pp. 869-870, 2003. 


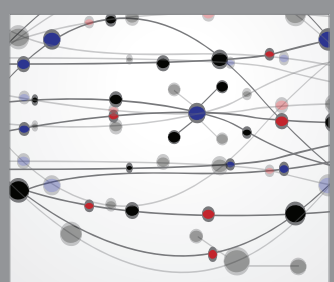

The Scientific World Journal
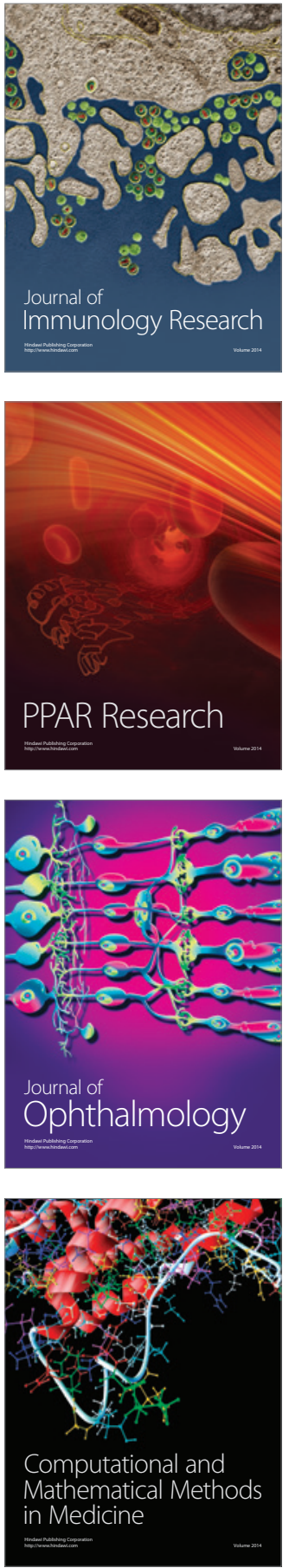

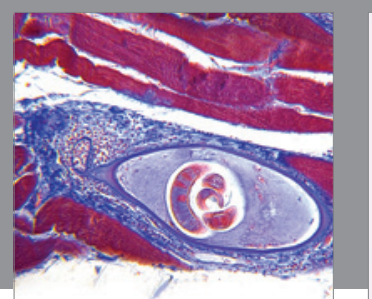

Gastroenterology Research and Practice

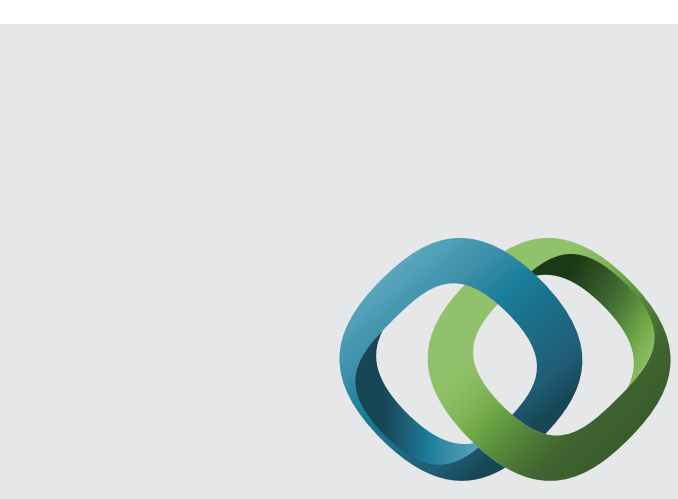

\section{Hindawi}

Submit your manuscripts at

http://www.hindawi.com
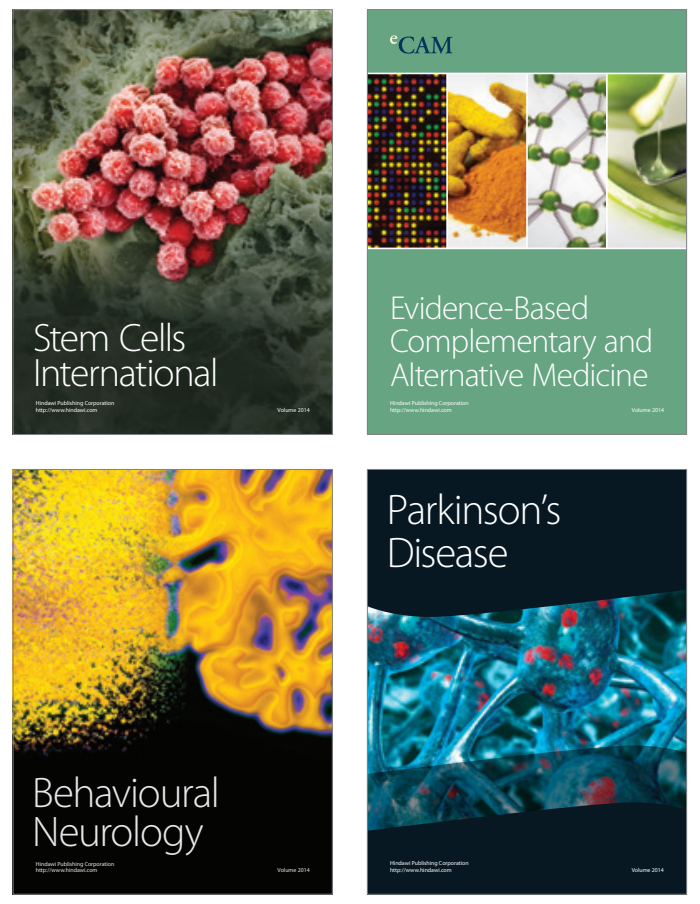
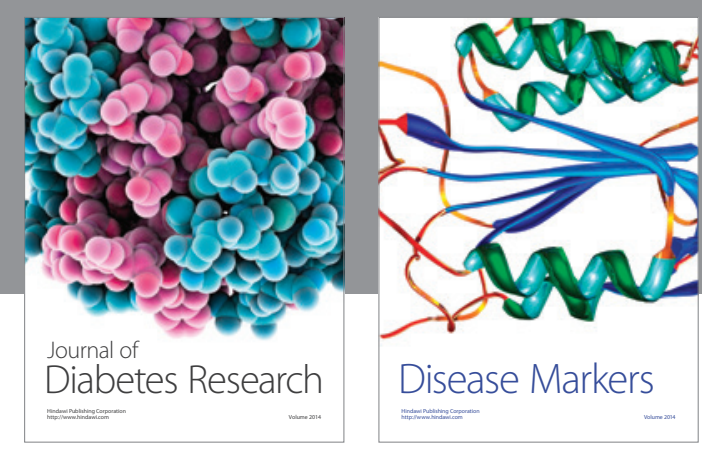

Disease Markers
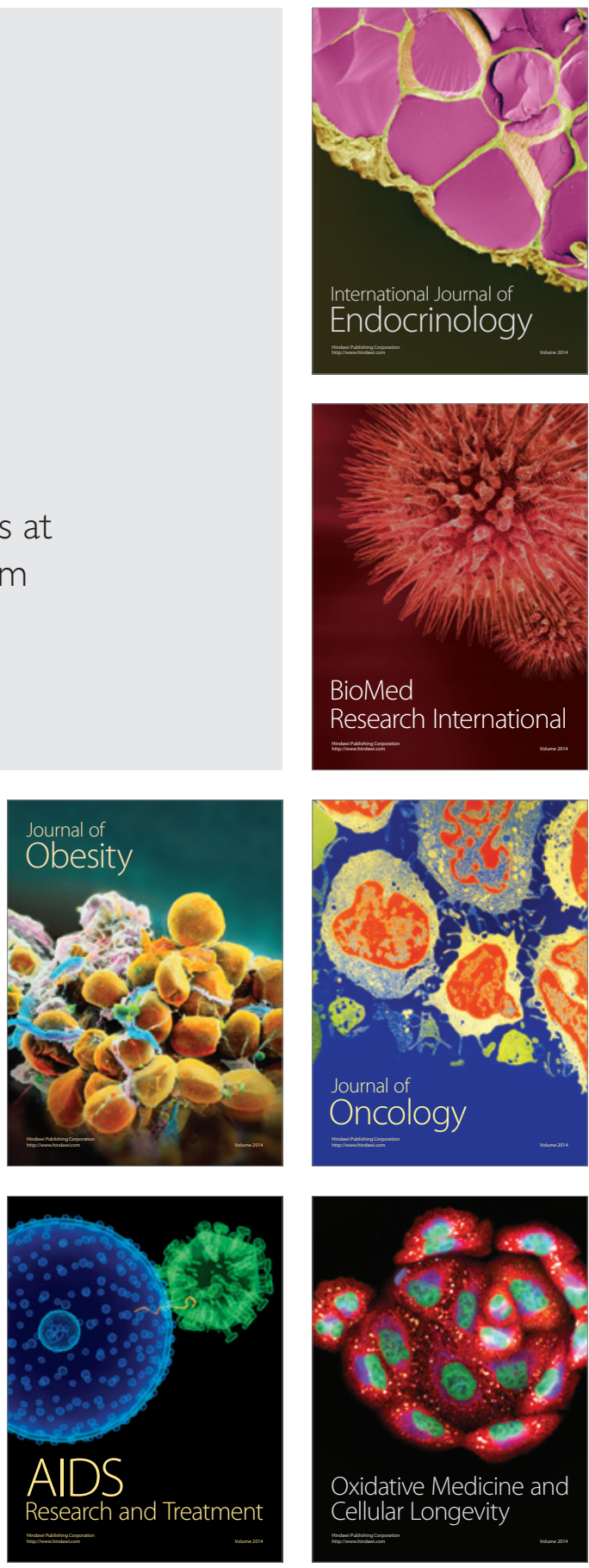